\title{
Research on the Approach to Describe Scenarios Based on Refinement
}

\author{
LI Yue-xia \\ College of Information Engineering \\ Qingdao University \\ Qingdao, China \\ liyuexiade@163.com
}

\begin{abstract}
Because of the lack of research on the abstraction of scenarios in the traditional method based on scenarios, and lack of an effective method to describe scenarios. In view of this, a method was proposed to describe scenarios based on refinement. First, a new scenario metamodel based on refinement relationship was proposed to structure scenarios. According to Rolland, scenarios were divided into different abstraction levels. And a high level of abstraction scenario was refined layer by layer into some low level of abstraction scenarios through refinement technology. Finally, an example was used to describe the process of this method. The approach can meet the needs of different stakeholders and help the requirement analysis of large and complex systems.
\end{abstract}

Keywords-software requirements; scenarios; metamodel; abstraction; refinement

\section{INTRODUCTION}

With the development of computers, the role of requirements engineering gained more and more attention. In the software development process analysis and description of requirements was increasingly important to its success, and is largely related to the quality of software products. Therefore, a complete and accurate description of requirements is essential. With the development of the requirements engineering, now there are several methods to describe the requirements, and in which the scenario is more effective method. Scenarios has a better intuitive, easy to communicate between the user and the software developer, and is suitable for describing system behavior and functional of application software.

Although there have get a lot of success, but the following key issues still exist in theory and in practice: (1) Lack of research different levels of abstraction of scenarios and relationships. (2) The lack of an effective method to describe scenarios.

To solve these problems, we propose a method to describe scenarios based on refinement. In this method, the abstraction level of scenarios is divided into three types: types, instance, and mixed. Instance scenarios, also called concrete scenarios, refer to specific agent names or events with concrete argument values. Type scenarios, also called abstract scenarios, are more abstract than instance scenarios. The entities described are not

\author{
WEI Chang-jiang \\ College of Information Engineering \\ Qingdao University \\ Qingdao, China \\ chj_wei@163.com
}

entity instance, like specific agent names, but entity types, like subscriber or customer.

The main innovations are:

(1) Constructed scenario metamodel based on abstraction and decomposition.

(2) Give the definition of scenarios and activities based on the relationship between scenarios and activities.

(3) Descriptions of the scene hierarchical method was proposed, and through examples detailed explain the process of hierarchical description of the scenarios.

\section{THE ANALYSIS OF SCENARIOS METAMODEL}

Because of different authors use the different definition of the scenarios in scenario-based requirements engineering, and the scenarios are usually constructed through metamodel. So there have a variety of scenarios metamodel.

\section{A. Scenarios metamodel from Rolland}

Rolland ${ }^{[1]}$ define a scenario as "composed of one or several actions, the combination of actions in a scenario describes a unique path leading from initial to final states of agents., ${ }^{\text {[2] }}$ Based on this definition, their scenario metamodel is showed in fig 1 . The concepts are showed in table 1.

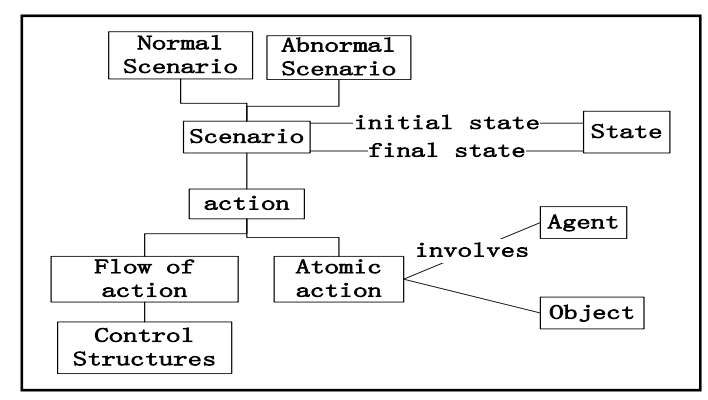

Fig.1. Scenarios metamodel from Rolland 
TABLE 1. The concepts of the scenario metamodel from Rolland

\begin{tabular}{|c|l|}
\hline \multicolumn{1}{|c|}{ Concepts } & \multicolumn{1}{c|}{ Explanation } \\
\hline normal and exceptional & $\begin{array}{l}\text { The former leads to the achievement of its associated } \\
\text { goal whereas the latter fails in normal goal } \\
\text { achievement. }\end{array}$ \\
\hline $\begin{array}{c}\text { initial state and final } \\
\text { state }\end{array}$ & $\begin{array}{l}\text { The initial state attached to a scenario defines a } \\
\text { precondition for the scenario to be triggered. A final } \\
\text { state defines a state reached at the end of the } \\
\text { scenario. }\end{array}$ \\
\hline Actions & $\begin{array}{l}\text { Actions are of two types: atomic and flows of } \\
\text { actions. }\end{array}$ \\
\hline $\begin{array}{c}\text { Atomic actions and } \\
\text { flows of actions }\end{array}$ & $\begin{array}{l}\text { Atomic actions are interactions from one agent to } \\
\text { another agent that affects some parameter object. A } \\
\text { flow of actions can have any one of the following } \\
\text { semantics: sequence, alternative, repetition, and } \\
\text { concurrency. Alternative and repetition carry flow } \\
\text { conditions and characterize the course of actions of } \\
\text { the scenario. }\end{array}$ \\
\hline Agent and objects & $\begin{array}{l}\text { An agent and resource objects may participate into } \\
\text { several atomic actions. }\end{array}$ \\
\hline
\end{tabular}

\section{B. Scenarios metamodel from Sutcliffe}

Sutcliffe ${ }^{[3]}$ defined a scenario as "one sequence of events that is one possible pathway through a use case." This definition suggests that many scenarios may be specified for one use case and each scenario represents an instance or example of events that could happen within a use case. Each scenario may describe both normal and abnormal behavior. Based on this definition, their scenario metamodel is showed in fig 2 . The concepts are showed in table 2.

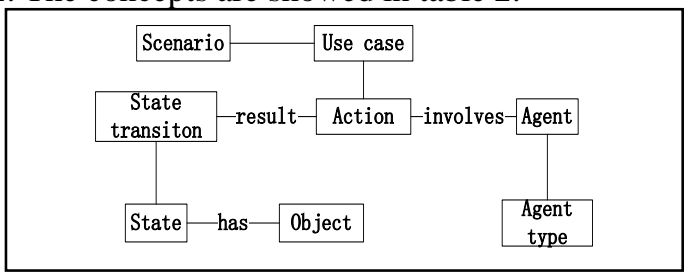

Fig.2. Scenarios metamodel from Sutcliffe

TABLE 2. The concepts of the scenario metamodel from Sutcliffe

\begin{tabular}{|c|l|}
\hline Concepts & \multicolumn{1}{c|}{ Explanation } \\
\hline scenarios & An instance or a example of use case. \\
\hline use case & $\begin{array}{l}\text { The use case specifies a network of actions linked to } \\
\text { the attainment of a goal which describes the purpose of } \\
\text { the use case. }\end{array}$ \\
\hline action & Each action involves one or more agents. \\
\hline agent & $\begin{array}{l}\text { Each agent can be either human, machine, composite or } \\
\text { an unspecified type. }\end{array}$ \\
\hline object & $\begin{array}{l}\text { Structure objects are persistent real world objects that } \\
\text { have spatial properties and model physical and logical } \\
\text { units of organization. }\end{array}$ \\
\hline
\end{tabular}

\section{Structuring scenarios base on refinement relationship}

Literature [4] defined a scenario as: Scenario describes a specific example of a goal (or a group of target) is satisfied or not satisfied. Scenario also can be defined as "a purposeful interaction maybe occurs between a few objects". It consists of one or more actions and each action represents an interaction between an object and the other objects and describe a unique path with a combination of a series of acts in a scenario ${ }^{[5] 6]}$.

The scenario uses a series of specific interaction sequences between the system and the user to illustrate how objectives are being satisfied. The goal achieved by activities is a subgoal of the goal achieved by scenarios. In addition, the active node is an organizational unit of activities and enlarges it you will find another activity diagram. And this activity diagram also can be described as a scenario. In this regard, this paper gives the definition of scenarios and activities:

The scenario defined as "a sequence of actions or activities maybe occurs between agents and this sequence of actions or activities can satisfy a goal."

Activity defined as "is a composition of actions or other activities and we will get another activity diagram to describe a scenario when you enlarge it."

A system's requirements should be described by a system of scenarios, rather than just one single scenario. And different stakeholders concerns different level of abstraction of requirements. So it is necessary to consider the level of abstraction of the scenarios, and can be transformed from high abstract to low. So this paper constructs a new scenario metamodel based on refinement of activity. As showed in fig3.

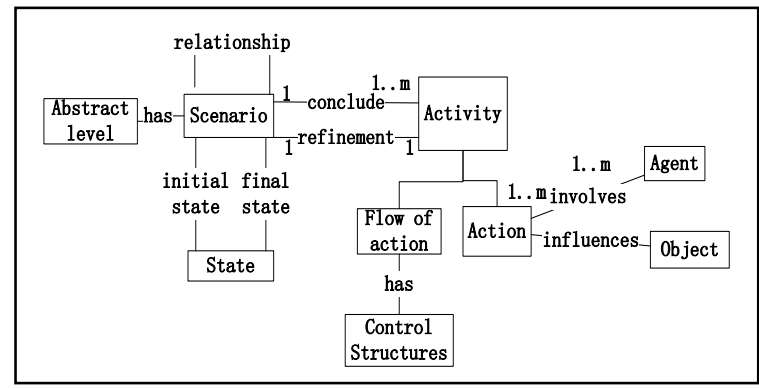

Fig.3. Scenario metamodel based on refinement

\begin{tabular}{|c|l|}
\hline Concepts & \multicolumn{1}{|c|}{ Explanation } \\
\hline State & $\begin{array}{l}\text { The initial state attached to a scenario defines a } \\
\text { precondition for the scenario to be triggered. A } \\
\text { final state defines a state reached at the end of the } \\
\text { scenario. }\end{array}$ \\
\hline Abstract level & $\begin{array}{l}\text { The content of a scenario can be concrete, abstract } \\
\text { or a mix of different degrees of abstraction or } \\
\text { concreteness. The three attributes of the } \\
\text { abstraction facet, namely Instance, Type and } \\
\text { Mixed, allow us to measure the level of abstraction } \\
\text { or concreteness suggested in the contents } \\
\text { description of a scenario-based approach. }\end{array}$ \\
\hline Activity & $\begin{array}{l}\text { Activities are if two types: atomic and flows of } \\
\text { actions. Scenario contains one or more activities } \\
\text { and Activities are the elements of the scenario. } \\
\text { activity of high level scenarios can be refined into } \\
\text { a low-level scenario }\end{array}$ \\
\hline Action & $\begin{array}{l}\text { Atomic actions are interactions from one agent to } \\
\text { another agent that affects some parameter object. }\end{array}$ \\
\hline between scenarios & $\begin{array}{l}\text { A flow of actions can have any one of the } \\
\text { following semantics: sequence, alternative, } \\
\text { repetition, and concurrency. }\end{array}$ \\
\hline A flow of actions & $\begin{array}{l}\text { The structural relationship between the scenarios } \\
\text { are: refinement, order (AND)and select (OR). }\end{array}$ \\
\hline &
\end{tabular}




\section{THE METHOD TO DESCRIBE SCENARIO BASED ON REFINEMENT}

In this method, scenarios were divided into different abstraction levels. And a high level of abstraction scenario was refined layer by layer into some low level of abstraction scenarios through refinement technology.

Different stakeholders concerns different level of abstraction of requirements. Therefore we need to describe requirements from different abstraction levels. In addition, it is difficult to describe all the system requirements just in a single scenario for a large complex system. So a system's requirements should be described by a system of scenarios. An effective structured approach to control the complex is layering technology, with a hierarchical scenario graph to decompose the complex. The type scenario is used to describe function of coarse-grained, and then use the decomposition technique to increase details for the type scenario, and refine it layer by layer. Layered realized abstraction and information hiding, which is not considered in the type scenario.

A system of a hierarchical scenario graph including the type scenario graph, mix scenario graph and instance scenario graph. Type scenario graph has only one scenario that describes the boundaries of the entire system. Mixed scenario graph is come from the refinement of activity elements in the type scenario, and its activity element can also continue to be refined. When the refinement is carried out until the activity of each scenario is atomic action, we will get a set of instances scene graph. Activity elements of instance scenario are atoms, and it cannot be decomposed. Obviously, this hierarchical scenario graph is a good method to understand and express system. Fig 4 depicts the process of refining scenarios. The figure also shows the relationship between scenarios. The refinement relationship relates a higher-level scenario to a lower-level scenario. Under this relationship, higher-level(less detailed) scenarios are refined into lower-level (more detailed) scenarios. The AND relationship between any two scenarios at the same level specifies a composition relationship between the two. The OR relationship between any two scenarios at the same level represents an alternative relationship between the two.

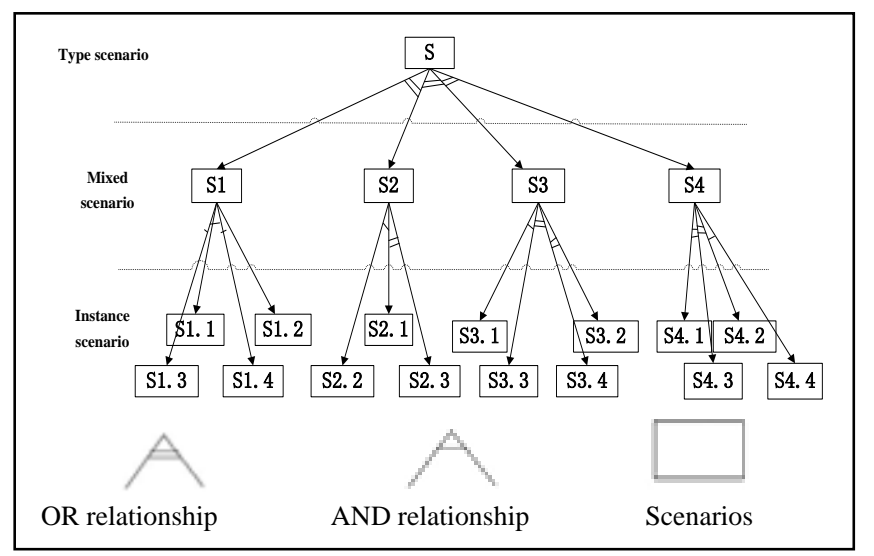

Fig.4. The refinement relationship between scenarios

The process of construct a hierarchical scenario graph is a structured system modeling process. This paper uses the example of ATM system to aid our description and illustration of this method. According to Rolland [7] and our above description, we classified ATM system scenario into contextual, system interaction and system internal scenarios. Fig.5 depicts such a hierarchy of scenarios for the ATM system.

For brevity, here we only illustrate the transformation of the Withdraw Cash scenario. Fig.6 depicts the process.

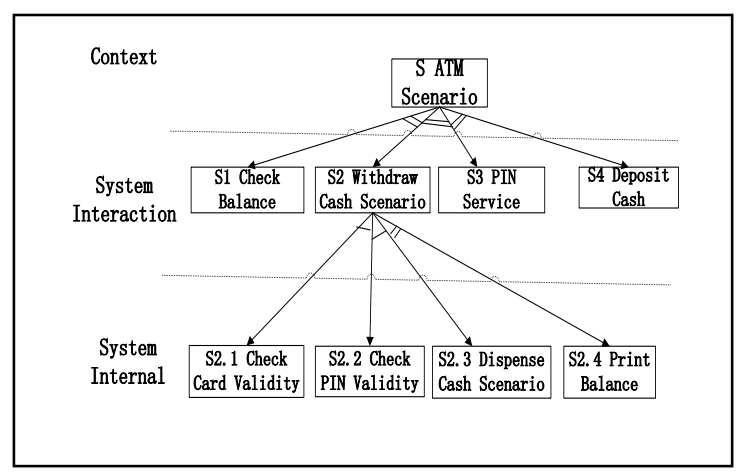

Fig.5. The refinement of ATM system

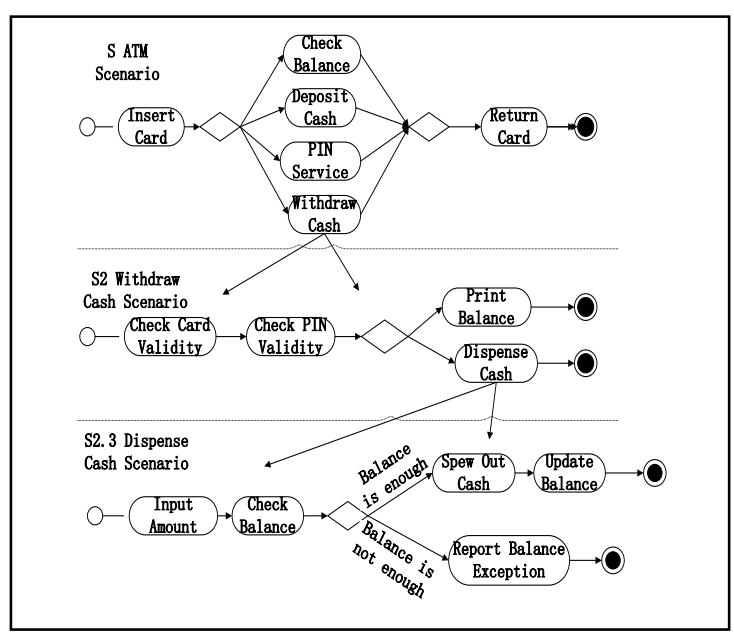

Fig.6. The refinement of the Withdraw Cash Scenario

For users, they are only concerned with what services ATM can provide for them, without having to understand how to achieve it. Therefore, the ATM system scenario abstract business layer to describe the requirement for ATM machine users, and helping users to understand.

For developers, the more detailed requirements to help them achieve this software.

For analysts, this method can help them to describe requirements very clearly and straightforward, and helps them to communicate with users and developers.

\section{CONCLUSIONS}

The proposed method can meet the needs of different stakeholders, and contribute to the requirements description of large and complex systems. Based on the current requirements engineering based on scenarios described has some drawbacks, caused by lack of description of the abstract level. The paper 
has argued that a remedy for these drawbacks is to propose a method to describe requirements based on refinement relationship. Finally, this paper uses the example of the ATM system to explain and illustrate the process of refinement. Our future work will find out how to achieve the refinement of scenarios.

\section{References}

[1] C.Rolland, C.Souveyet, CB.Achour. Guiding goal modeling using scenarios. IEEE Transactions on Software Engineering, 1998b,24 (12):1055-1070.
[2] DaiJuan. Research on the Goal and Scenario Based Requirements Analysis Technique with Use Case Driven. Xi'an : Xidian University,2012.

[3] Sutcliffe Alistair, Scenario-based requirements analysis. Requirements Engineering , 1998,3 (1): 48-65.

[4] Klaus Pohl. Requirements engineering: basic, principle and technology. Beijing : China Machine Press,2012.

[5] Zhou Ting. Requirements Engineering with Scenario Driven.Changsha: National University of Defense Technology,2002.

[6] Ling Chen. Research on MIS requirement engineering based on scenario description.Shanghai: Tongji University,2007.

[7] C.Rolland , CB.Achour, C.Cauvet and so on. A proposal for a scenario classification framework. Requirements Engineering Journal 1998a ,3 (1), 23-47. 